\title{
Seminal fluid proteins induce transcriptome changes in the Aedes aegypti female lower reproductive tract
}

\author{
I. Alexandra Amaro', Yasir H. Ahmed-Braimah², Garrett P. League', Sylvie A. Pitcherr', Frank W. Avila ${ }^{3}$, \\ Priscilla C. Cruz ${ }^{1}$, Laura C. Harrington ${ }^{1 *}$ and Mariana F. Wolfner ${ }^{4^{*}}$
}

\begin{abstract}
Background: Mating induces behavioral and physiological changes in the arbovirus vector Aedes aegypti, including stimulation of egg development and oviposition, increased survival, and reluctance to re-mate with subsequent males. Transferred seminal fluid proteins and peptides derived from the male accessory glands induce these changes, though the mechanism by which they do this is not known.

Results: To determine transcriptome changes induced by seminal proteins, we injected extract from male accessory glands and seminal vesicles (MAG extract) into females and examined female lower reproductive tract (LRT) transcriptomes $24 \mathrm{~h}$ later, relative to non-injected controls. MAG extract induced 87 transcript-level changes, 31 of which were also seen in a previous study of the LRT $24 \mathrm{~h}$ after a natural mating, including 15 genes with transcript-level changes similarly observed in the spermathecae of mated females. The differentially-regulated genes are involved in diverse molecular processes, including immunity, proteolysis, neuronal function, transcription control, or contain predicted small-molecule binding and transport domains.
\end{abstract}

Conclusions: Our results reveal that seminal fluid proteins, specifically, can induce gene expression responses after mating and identify gene targets to further investigate for roles in post-mating responses and potential use in vector control.

Keywords: Aedes aegypti, Seminal fluid proteins, RNA-Seq, Post-mating responses, Immunity, Proteolysis, Transcriptional control

\section{Introduction}

Aedes aegypti is a significant mosquito vector of the viruses that cause Zika (ZIKV), chikungunya (CHIKV), yellow fever (YFV), and dengue (DENV) [1-4]. There are no approved anti-viral therapies for Aedes-borne pathogens $[5,6]$ and while an effective vaccine for yellow fever exists, supplies have become depleted in recent outbreaks

\footnotetext{
*Correspondence: Ich27@cornell.edu; mfw5@cornell.edu

1 Department of Entomology, Cornell University, Ithaca, NY 14853, USA

${ }^{4}$ Department of Molecular Biology and Genetics, Cornell University,

Ithaca, NY 14853, USA

Full list of author information is available at the end of the article
}

[7]. A commercially licensed vaccine for dengue has limited efficacy and is not widely used [8]. Efforts to reduce disease burden rely on vector control. However, conventional mosquito control measures using insecticides can be operationally difficult and ineffective in areas where insecticide resistance is a major problem [9], and may have detrimental effects on beneficial insects [10]. Population replacement or reduction control strategies that involve deployment of modified mosquitoes show promise for control [11]. However, these approaches rely on successful mating and reproduction [12]. 
Mating in many insects, including Ae. aegypti, initiates behavioral and physiological changes in females (reviewed in [13]). Seminal fluid proteins (SFPs), which are primarily produced in the male accessory glands (MAG) as well as the ejaculatory duct, are transferred to females during mating and play a large role in inducing post-mating responses [13-15]. Much of the detailed work on the nature, function, and effects of SFPs has been conducted in Drosophila melanogaster (reviewed in [13]). While less is known about Aedes male accessory gland-derived SFPs, a recent analysis cataloged 280 SFPs in Ae. aegypti [16], similar to the number reported for D. melanogaster [17]. However, specific functionality for nearly all of these proteins has not been determined. Mating or injection of SFP-containing MAG extracts stimulates Ae. aegypti females to oviposit developed eggs several days after blood meal ingestion [18-22]. In addition, survival of mated and MAG extract-injected females is higher than virgins' irrespective of whether a blood meal was consumed [22], and females that mate with non-SFP-depleted virgin males survive longer [23]. Females are monandrous and demonstrate reduced mating receptivity after an initial mating [24, 25]. This effect sets in quickly, with only a quarter of females being receptive to re-mating within $2 \mathrm{~h}$ after the first mating event, and complete mating refractoriness is established within $24 \mathrm{~h}$ post-mating [26]. A low dose of MAG extract is sufficient to induce this effect $[25,27]$, which persists through multiple gonotrophic cycles [26]. While the transfer of male mature Head Peptide- 1 contributes to short-term transient refractoriness [28], the male proteins/peptides responsible for long term establishment over the duration of the female lifespan are unknown. MAG extract injection into a female, or mating, reduces the likelihood that the female and her potential mate will harmonize their flight tones [29], a mechanism by which SFPs could reinforce resistance to re-mating. It is likely that there are additional yet unknown effects of SFPs on female mosquito post-copulatory biology and behavior.

Knowing the molecular pathways that are activated in Ae. aegypti females post-mating would be helpful in guiding functional assays and ultimately understanding mosquito post-mating biology and vector control targets. However, these pathways are presently unknown. Transcriptome analysis, first with microarrays and now with Next Generation Sequencing (NGS), has been a valuable tool to examine gene expression changes in whole bodies or specific tissues of mated females in a variety of insect orders, including Diptera (Drosophilidae, Tephritidae, Aleyrodidae, Culicidae), Hymenoptera, Lepidoptera, and Coleoptera [30-45]. We previously examined transcriptlevel changes in the Ae. aegypti female lower reproductive tract (LRT, which consists of the bursa, spermathecae, and oviducts) at 0,6 , and $24 \mathrm{~h}$ after mating and identified transcriptional changes in protease and antimicrobial peptide genes as well as molecules that regulate immunity [46]. Similar analysis of various reproductive tissues minus the ovaries in Anopheles gambiae and D. melanogaster have identified changes in transcripts associated with metabolic function, catalytic activity and immunity [47-50]. Identification of a cluster of genes that respond to mating in An. gambiae, all linked by 20E hormonal regulation, led to the discovery that $20 \mathrm{E}$ was an activator of post-mating responses in that mosquito [49]. This work demonstrated the power of transcriptome analysis to provide insights into female reproductive pathways.

Specific female post-mating transcription changes are induced by different ejaculate components, as demonstrated in D. melanogaster where different sets of female genes are regulated by sperm, by SFPs or by the act of, or some other component of mating [31,33]. Transcript profiles of An. gambiae and Ceratitis capitata females after mating to sperm-less vs. wild-type males gave insights into sperm-specific female responses [51-53]. However, it is not known which genes in the Ae. aegypti female LRT respond specifically to seminal fluid proteins, the initiators of post-mating responses [13]. Identification of this subset of genes from among those in females that respond to mating will provide insight into the as-yet unknown mechanisms that underlie postmating responses. In D. melanogaster, SFPs transit into the hemolymph through damage to the female vaginal wall during copulation $[54,55]$ and injection of SFPs into Drosophila hemolymph causes post-mating-like changes [56]. As the injection of MAG extracts, but not saline, into Ae. aegypti virgin females also causes post-matinglike changes [22, 25, 27, 29], we utilized this means to introduce seminal fluid proteins into females to investigate their influence on transcript levels. Transcripts thus identified are regulated either directly or indirectly upon introduction of seminal proteins during a natural mating and identify female pathways regulated by SFPs that participate in post-mating responses.

\section{Results and discussion}

Saline injection into Ae. aegypti females induces expression of genes involved in wounding and immunity pathways in their LRTs

The female mosquito's lower reproductive tract (LRT) is critical to sperm storage, egg transit, and fertilization. These organs undergo transcriptome changes after mating $[46,57]$, presumably as part of the female's transition to high reproductive efficiency. To determine which of these changes are induced by seminal fluid proteins (SFPs), we examined transcriptome changes in the LRT at $24 \mathrm{~h}$, the time at which the greatest number 


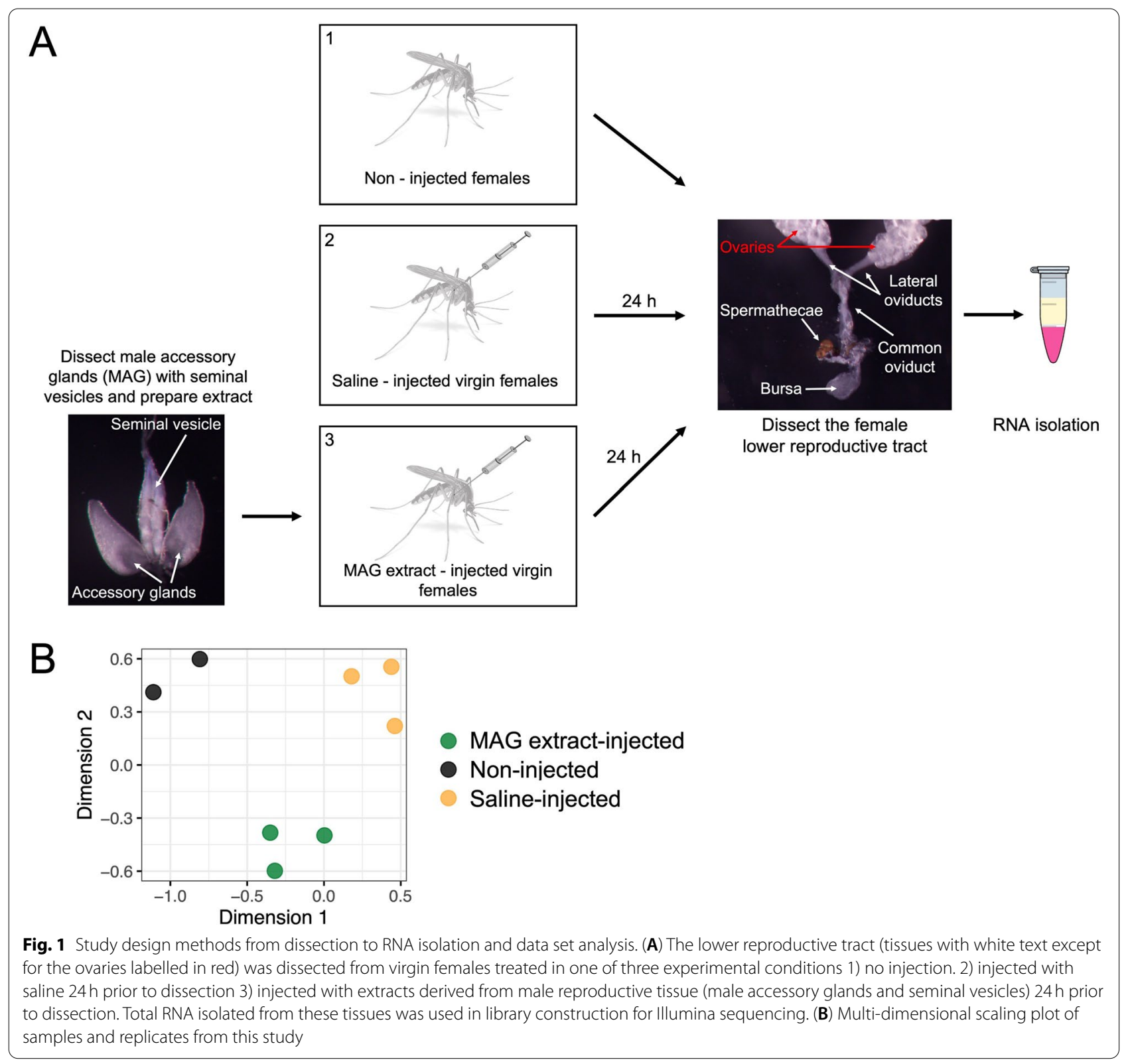

of transcriptional changes were observed in the female LRTs of mated Ae. aegypti [46] and An. gambiae [49], following injection of lysate derived from the male accessory gland (MAG), the primary tissue that produces SFPs, and associated seminal vesicles, hereafter referred to collectively as MAG extract (Fig. 1A). To identify transcriptome changes that were induced simply by needle-wounding and saline, we first compared the transcriptomes of the LRTs of saline-injected females with those of non-injected females. We observed differentially expressed genes (DEGs) at $24 \mathrm{~h}$ after saline injection, consisting of 10 down-regulated and 122 up-regulated genes. (Fig. 2A, Supplementary Table 1). As expected for a response to injection, DEGs with known or potential function in wounding cascades or immunity pathways in mosquitoes and other insects were represented $[58,59]$. These include up-regulation of RNAs encoding peroxidases (AAEL026038, AAEL004388, AAEL004386) and a dopachrome-converting enzyme (AAEL000064) that is involved in melanization pathways $[60,61]$. We observed up-regulation of RNAs encoding known or predicted serine protease domains (AAEL002686, AAEL007102, AAEL015533, AAEL015432, AAEL005748, AAEL002624, AAEL006576, AAEL024784), including CLIP-serine 


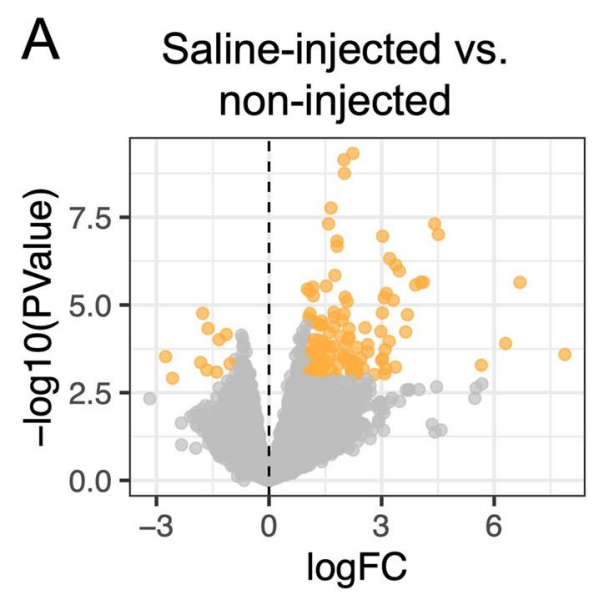

\section{B MAG extract-injected \\ vs. non-injected}

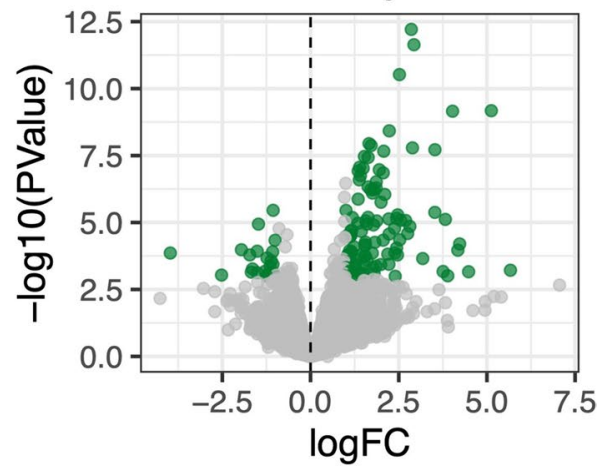

\section{MAG extract injected} vs. Saline-injected
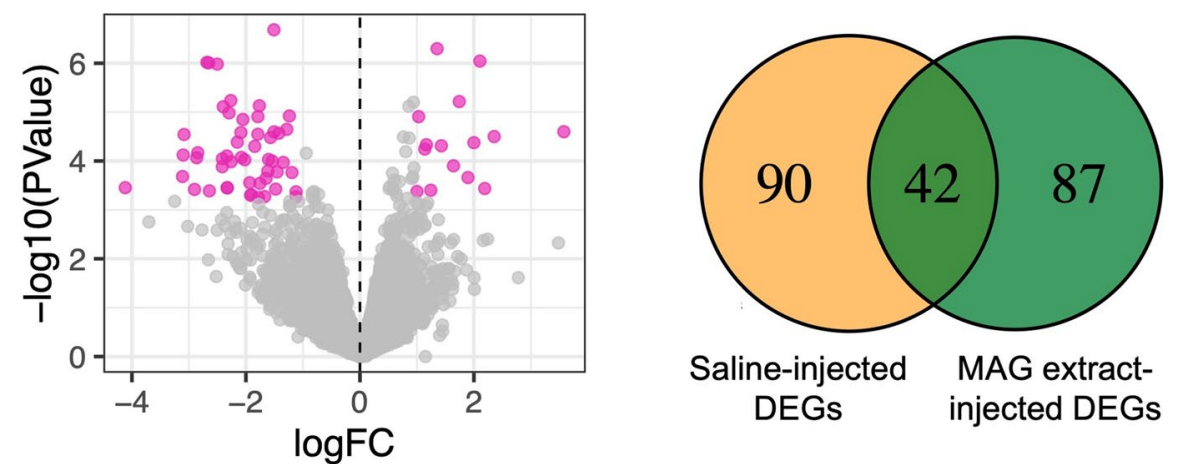

Fig. 2 Transcript-level changes observed in female LRTs $24 \mathrm{~h}$ after injection. Volcano plots depicting changes in transcript levels in (A) saline-injected females or (B) MAG extract-injected females each compared to non-injected females. (C) Transcript level comparisons between MAG extract-injected and saline-injected females are similarly depicted. Significant transcript level changes are highlighted $(P<0.05 ; F D R<0.05)$ in yellow, green, and pink. (D) Venn diagram comparing number of DEGs in saline- and MAG extract-injected over non-injected mosquitoes

proteases (AAEL010773, AAEL002124, AAEL000037, AAEL024669, AAEL002585), which together with a protein containing a serpin domain (AAEL020823) are classes of proteins known to have regulatory control over melanization, coagulation, and immune pathways [58, 62-65]. Additional up-regulated DEGs associated with the immune responses included those with C-Type lectin domains (AAEL011408, AAEL023353), a C-Type lysozyme (AAEL017132) and anti-microbial peptides (AMPs) Defensin C (AAEL003832), Defensin D (AAEL003857), and Holotricin (AAEL017536) [66]. The most up-regulated gene, AAEL008098/PIWI, is involved in RNA silencing as a part of the mosquito anti-viral response [67]. These changes in levels of transcripts with immune function in the LRT indicate a response to wounding from injection and introduction of saline into the thorax.
These results are consistent with other studies that have reported immune activation after injection either alone or with media, confirming the quality of our data [68-72]. Priming Ae aegypti immunity by needle injection with RPMI media leads to increases in relative expression of RNAs encoding the AMPs Defensin, as we also observed for the LRT, as well as Cecropin and Attacin [68]. Circulating hemocytes, immune cells in the hemolymph, that are isolated from Ae. aegypti adults following injection of saline show significantly increased protein concentrations when compared to hemocytes from non-injected adults [69], which may reflect activation as hemocytes are recruited to the site of injury. Differential expression in D. melanogaster of immune-related, cell proliferation, and cell migration genes is observed in third instar larvae hemocytes following needle puncture [70]. The injection set-up in our study does not lend itself to practically sterilize each needle, hence some of the observed DEGs 
could be in response to bacteria or other pathogens that were introduced either on the needle or by opportunistic entry into the wound site from the mosquito cuticle. In D. melanogaster, activation of cellular immune responses can be achieved by sterile wounding alone [71]. Clot formation due to wounding is not fully understood in mosquitoes, but electron microscopy of sterile needle damage in Armigeres subalbatus larvae demonstrates involvement of granulocytes and deposition of melanin [72], which is synthesized by peroxidases and dopachrome-converting enzyme under the regulatory control of serine proteases, each of which was observed to be up-regulated upon injection in our data. Injection into adults is not without consequence, and our dataset revealed these changes in an unbiased manner to include more than the best characterized immune and wounding responses. Seven of the ten down-regulated genes and 67 of the 122 up-regulated genes are currently uncharacterized in the current genome annotation and may define new players in responses to wounding or infection.

\section{MAG extracts induce genes in the LRT that are involved in a variety of molecular processes}

MAG extract injection down-regulated 21 genes and up-regulated 108 genes relative to non-injected females after 24h (Fig. 2B). To focus our analysis on genes regulated specifically by MAG contents, and not from the injection itself, we removed the 42 DEGs that were also observed in saline-injected females compared to noninjected from our 129 MAG extract-injected DEGs dataset (Fig. 2D, Supplementary Table 1). The remaining 87 genes (68 up-regulated, 19 down-regulated) show significant transcript-level changes in the LRT following MAG extract injection that are involved in a variety of molecular processes (Supplementary Table 2). We observed DEGs with functions in immunity pathways, including pattern recognition receptors such as C-type lectins (AAEL000543, AAEL014382, AAEL012353, AAEL026265), proteins with a peptidoglycan recognition protein domain (AAEL009474, AAEL019745), a gramnegative binding protein (GNBP) (AAEL007626), and macroglobulin (AAEL001794) which can complex with leucine-rich repeat containing proteins (AAEL001414) [58, 59]. MAG extract injection resulted in up-regulation of AAEL019728, a suppressor of cytokine signaling (SOCS) associated with Janus kinases/Signal transducer and activator of transcription protein (JAK/ STAT) pathways [73] and cecropins (AAEL029038, AAEL029044). Transcript levels were also altered for 11 genes with a predicted serine protease domain, which is involved in regulatory control of multiple cellular functions [58, 62, 64, 65]. Among other notable detected changes to transcripts were those in genes with predicted juvenile hormone binding protein domains (AAEL001323, AAEL009927), an odorant binding protein (AAEL006109), five genes with associated neuronal function (AAEL014450, AAEL019604, AAEL003413, AAEL023634, AAEL005945), and three genes involved in transcriptional control (AAEL004097, AAEL003861, AAEL013321) (Supplementary Table 2). In the current genome annotation, ten genes are annotated as non-coding RNAs, and we observed ten DEGs that are uncharacterized for which we could also not predict a conserved domain or find a similar alignment to known genes by BLAST.

\section{Transcript changes that are observed after both mating and MAG extract injection represent gene pathways that are influenced by seminal fluid proteins}

Physical interactions between males and females involved in mating, as well as the transfer of ejaculate comprising of not only sperm, but also SFPs and RNAs all contribute to transcriptional changes in mated females [31, 33, 46]. We previously examined transcriptional changes in response to mating after $24 \mathrm{~h}$ in the LRT [46]. To distinguish between transcript changes observed after mating that were due to SFPs, rather than those changes that result from other mating components, we compared DEGs identified after both MAG extract-injection and natural mating. Using an updated analysis of the DEGs at $24 \mathrm{~h}$ post-mating with a more recent release of the Ae. aegypti genome [74] (Supplementary Fig. 1, Supplementary File 1), we identified DEGs (10 down-regulated and 21 up-regulated) in common between MAG extractinjected and similarly-aged mated females (Fig. 3A, C, Supplementary Tables 2, 3). In particular for the similarly up-regulated genes, there was congruence between the magnitude of the transcript-level changes between the two studies. When the $\log \mathrm{FC}$ for these genes in each study were plotted together, those genes clustered around the line of equality which represents an equal log foldchange from each study. Futhermore, these commonly altered genes included one down-regulated gene and 5 up-regulated genes that responded to injection of both MAG extracts and saline (Fig. 3A, C, Supplementary Table 3). These 6 genes have functions consistent with immunity and injury pathways, such as defensins and serine proteases, which could explain the presence of these DEGs in saline-injected females. Overall, the 31 overlapping genes are those that are likely to be influenced by SFPs during mating, rather than sperm or mating behavior, and can give insights into important post-mating pathways in females. The DEGs have functions in multiple pathways as discussed below. 

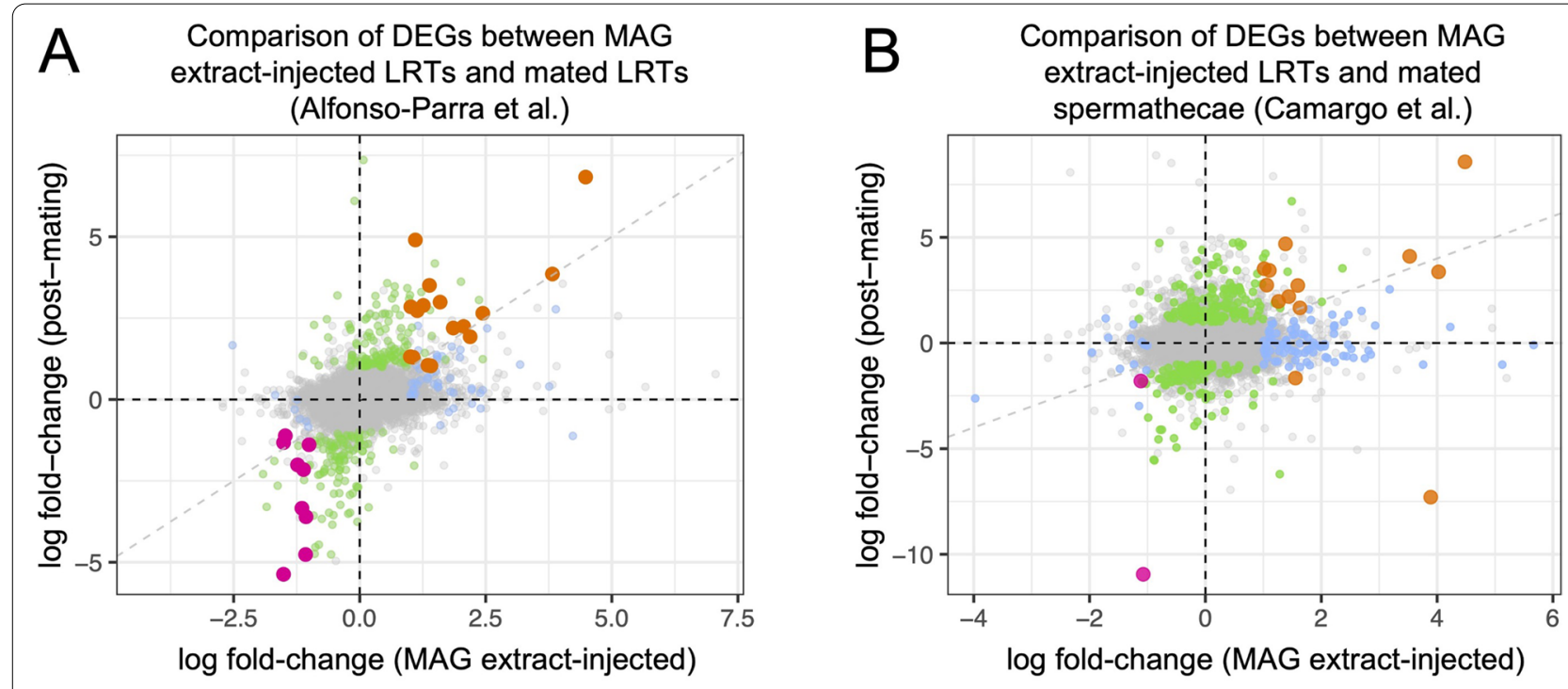

C

Saline-injected vs. non-injected (LRT)

Mated vs. virgin (LRT)

(Alfonso-Parra et. al)

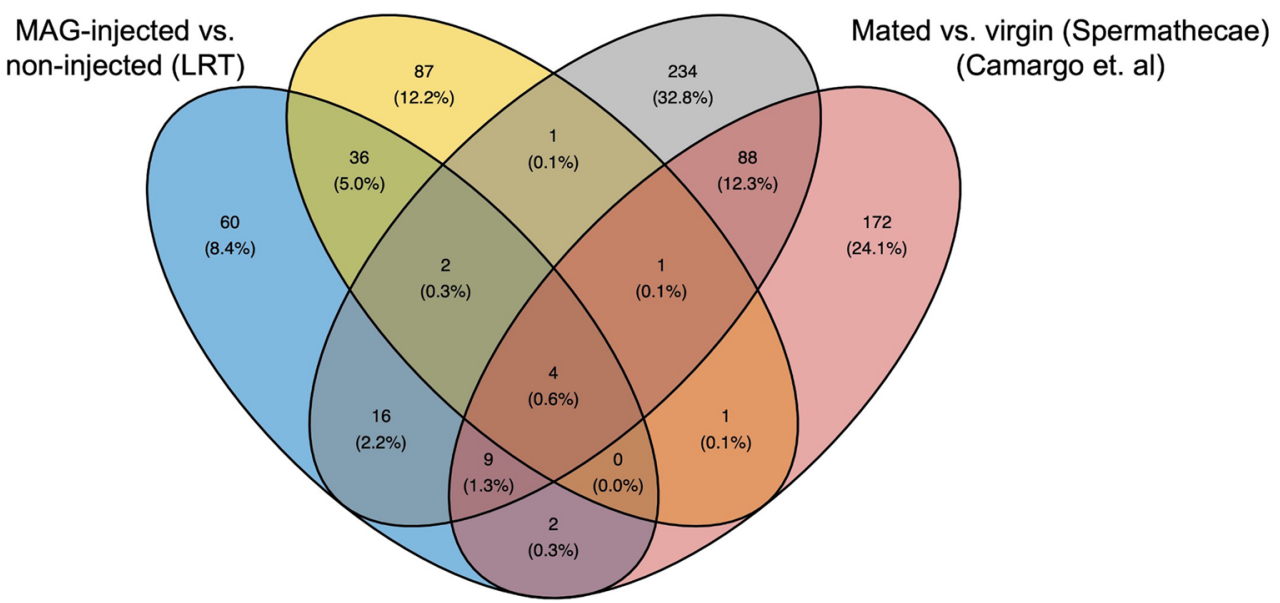

Fig. 3 Female reproductive tissues injected with MAG extract or naturally mated after $24 \mathrm{~h}$ share DEGs. Comparison between DEGs in the (A) LRT [46] or (B) spermathecae [57] of mated females (logFC in relation to virgin females on y-axis with significant DEGs noted in green) and LRT of MAG extract-injected females (logFC in relation to non-injected females on $x$-axis with significant DEGs noted in blue). Dark orange dots denote common up-regulated genes in both studies. Magenta dots denote common down-regulated genes. The dashed diagonal line represents equal log fold-change for genes in the two treatment groups. (C) Venn diagram illustrating the DEGs (compared to non-injected or virgins) from four treatment groups

\section{Transcripts of genes with immune function}

We observed changes in LRTs from mated and MAG extract-injected females of RNAs from genes with known or predicted immune function (AAEL000200, AAEL001794, AAEL020192, AAEL007626, AAEL029044, AAEL019728, AAEL003832, AAEL003857) [59]. Expression of these genes was not induced by needle puncture and saline alone, because when we compared transcript levels in MAG extract-injected versus saline-injected LRTs (Fig. 2C, Supplementary Table 2), we found significantly higher transcript levels for AAEL003832/ Defensin C (logFC 1.43), AAEL029044/predicted Cecropin ( $\operatorname{logFC} 1.90$ ), and AAEL005641/C-Type lectin ( $\operatorname{logFC} 2.35)$, indicating that induction of these genes is above what would be seen for injury alone and could reflect additional up-regulation due to SFPs. Additionally, we observe a statistical enrichment of DEGs with Gene Ontology (GO) terms corresponding to immune functions (Supplementary Fig. 2). This enrichment was not observed with DEGs from saline-injected females, 
indicating that transcriptional changes in a number of immune genes are the result of introduction of MAG extract components, not from injury due to injection. Although the MAG tissue was immediately removed to fresh saline prior to homogenization, males were not surface-sterilized prior to dissection. Thus, we cannot exclude the small possibility that some microbial contaminants from the carcass could be in our extract and contribute at some level to induction of immune response-genes in females. Similar mating-induced changes in immune gene transcript levels have also been reported in mated Drosophila females [30, 31, 45, 47, 48, 75-78], including a recent analysis finding distinct mating-induced transcriptional changes in immune genes in the different tissue types that comprise the D. melanogaster female reproductive tract [50]. Other insects demonstrate changes in immune gene transcript levels after mating, including An. gambiae [49], Bactrocera tryoni [41], Bemisia tabaci [44], ants [79], and Bactrocera dorsalis [39]. The overall significance of differential expression of immune genes after mating is the subject of continued study [80-83].

Recent work has highlighted roles for immune genes in reproductive pathways. For example, C-type lectins, such as AAEL005641/C-Type lectin which was one of the DEGs in our dataset, have been classically associated with immune function, yet in Ae. aegypti knockout of C-type lectin GCTL-3 using CRISPR decreased dengue virus susceptibility, but also altered germ line development and reduced fertility [84]. The complement-like molecule Thioester-containing protein 1 (TEP1) in $A n$. gambiae recognizes malaria ookinetes and also clears damaged sperm from the testes [85]. While we did not observe the Ae. aegypti homolog of TEP1 as a DEG responsive to MAG extract injection, we did observe a macroglobulin/complement gene to be differentially regulated by MAG extract (AAEL001794). It will be interesting to investigate the immune genes influenced by SFPs uncovered here for roles in reproduction, particularly the Suppressor of Cytokine Signaling (SOCS) gene's involvement in JAK/STAT immune signaling pathways [73].

\section{Transcripts encoding predicted proteases}

Proteins with serine protease function (AAEL001178, AAEL010867, AAEL014567, AAEL015533, AAEL000037) shared between MAG extract-injected and mated LRTs are involved in a variety of cellular processes, including immunity pathways as discussed previously, but also in reproduction. Serine proteases comprise a large fraction of proteins found in the seminal fluid of both insects and mammals (reviewed in [86]). These proteins contribute to sperm maturation, maintenance, and release, with multiple proteases acting to release sperm from the seminal clot in humans, as well as promotion of egg laying in insects [86-89]. Two serine proteases in the $A n$. gambiae female reproductive tract have roles in normal processing of the mating plug and subsequent release of the hormone 20E, a regulator of post-mating responses in An. gambiae [90]. Importantly, proteases can facilitate amplification of a pathway via signaling cascades with sequential activation of multiple proteases by cleaving propeptides [86]. AAEL014567/Oviductin has two predicted serine protease domains. Oviductins in the oviduct of Xenopus laevis and Bufo japanicus cleave egg envelope glycoproteins to facilitate fertilization [91, 92].

\section{Transcripts encoding proteins with predicted small molecule binding or hormone transport domains}

AAEL001323 and AAEL006109/OBP23, which were similarly up-regulated in MAG extract-injected and mated females, have predicted functions in small molecule or hormone transport. AAEL001323 has a predicted hemolymph juvenile hormone-binding protein domain, and shares BLAST alignment with the takeout family of genes involved in metabolism, aging, and courtship of males [93]. The highest observed up-regulated transcript in response to both MAG extract injection and mating is AAEL006109/OBP23, a member of the odorant binding protein class [94]. OBP23 is expressed in a variety of tissues, such as male antennae and female rostrums $[95,96]$ and is up-regulated in the midgut after Zika infection in Ae. aegypti (Maria Onyango, personal communication). OBPs have been identified in the reproductive tracts of male and female insects (reviewed in [97]), including the moth Athetis dissimilis [98], and the seminal fluid of Drosophila [99] (reviewed in [100]). The role of odorant binding proteins in reproduction is not clear, but they might potentially act as carriers of pheromones transferred to females during mating.

\section{Transcripts encoding gene-regulatory proteins}

Transcription factors can stimulate or repress gene expression in pathways that are important for fertility, thus amplifying initial mating signals and potentially leading to post-mating changes. We identified two down-regulated genes with domains involved in gene expression regulation in both MAG extract-injected and mated LRTs. AAEL004097/Hairy \& Enhancer of Split has a helix-loop-helix orange (HLH-O) domain. Proteins containing this domain are implicated in mosquito and Drosophila embryogenesis [101]. Knockdown of HLH-O domain genes in red flour beetle Tribolium castaneum revealed roles for these proteins in female survival, reproduction, and embryogenesis [102]. AAEL003861/ BMP-induced factor has a BTB/POZ domain (Broadcomplex, Tramtrack \& bric-a-brac/poxvirus \& Zn finger) 
which has characterized roles in development [103]. For example, the Drosophila BTB/POZ-containing protein MAMO, located in germ cells, activates factors required for completion of meiosis in order to successfully generate zygotes [104]. Additional analysis of the upstream genomic regions of SFP-responsive DEGs could reveal shared binding motifs for transcription factors regulating post-mating responses, but a larger sample of DEGs than we detected here would be needed to increase the power of the algorithms used to predict these sites.

\section{Additional MAG extract-responsive transcripts}

Other DEGs observed in LRTs from both MAG extractinjected and mated females have a variety of predicted functions. This includes up-regulation of AAEL019604, a gene that shares sequence alignment with a synaptic vesicle membrane protein, and down-regulation of AAEL014450, a peptidyl-glycine alpha-amidating monooxygenase which generates alpha-amidated peptides. Amidation of $\mathrm{C}$-terminal amino acids is required for activity of hormones and neuropeptides. We previously reported the up-regulation of RNAs encoding transferrin after mating [46] and observed a similar increase in transcript levels after MAG extract injection. LRTs from MAG extract-injected and mated females had lower transcript levels of AAEL008342, which encodes a monocarboxylate transporter. Monocarboxylate transporters were also down-regulated in female honeybees injected with seminal fluid when compared to buffer-injected [105]. Down-regulation of oxidoreductase (AAEL09685) and up-regulation of AAEL012382, which contains a predicted C-terminal nucleosidase domain, and of AAEL014719, an inosine-uridine preferring nucleoside hydrolase, were also detected. Three down-regulated RNAs (AAEL023640, AAEL024292, AAEL022727) and one up-regulated RNA (AAEL026265) from both mating and MAG extract-injection are annotated as noncoding RNAs. The term non-coding RNA encompasses multiple species of RNA. The four ncRNAs we detected are all longer than $200 \mathrm{bp}$, consistent with what would be considered long noncoding (lncRNAs) (reviewed in [106]). Studies in vertebrates and invertebrates have demonstrated that lncRNAs have regulatory roles in gene expression in a variety of cellular and biological processes (reviewed in $[106,107])$. Thus, the putative lncRNAs we identified could be interesting targets to explore for potential roles in modulating post-mating responses.

In summary, identification of common DEGs from both studies demonstrate that MAG extract injection can recapitulate some gene-expression changes in the LRT observed after mating.

\section{Comparison of DEGs from MAG extract-injected LRTs with those in spermathecae from mated females identify spermathecal transcript changes that are responsive to seminal fluid proteins}

There are post-mating transcriptome changes in $A e$. aegypti spermathecae, the sperm storage organs within the female LRT [57, 108]. Spermathecae store and maintain sperm, making this tissue vital for successful reproduction, particularly in monandrous mosquito species where stored sperm fertilize eggs for the remainder of a female's life (up to 100 days or more [87]). We compared our MAG extract-injected LRT DEGs with genes recently reported in Ae aegypti to be differentially expressed in the spermathecae of nonblood fed females at 24h after mating [57] (Fig. 3B, C, Supplementary Tables 2, 3). We found 15 genes that overlapped between spermathecal tissue from mated females and LRTs from MAG extract-injected females (LRT includes the spermathecae). These included DEGs involved in immune pathways (AAEL019728), oxidative stress (AAEL009685), peptidase function (AAEL010867, AAEL014567, AAEL022646), neuronal function (AAEL014450, AAEL019604) and small molecule/hormone transport (AAEL001323, AAEL006109). These are consistent with protein classes identified in the spermathecae of Ae. aegypti after mating [57, 108], and also from transcriptional analysis of sperm storage organs after mating in Drosophila [109], An. gambiae [53], and ant queens [110]. Similar to what was found in the comparison of MAG extract-injected with mated LRTs (Supplementary Table 3), four of the overlapping genes (AAEL009291, AAEL000037, AAEL003832, AAEL003857) were also differentially expressed with saline injection and have functions consistent with immune pathways. Furthermore, 13 of the 15 overlapping genes, including all four that were also induced by saline, were identified as DEGs at $24 \mathrm{~h}$ in the mated LRT [46], and 14 of the 15 were similarly altered in the spermathecae of mated females 7 days post-eclosion [108], demonstrating consistency in our results. MAG extract-injected DEGs that overlap with transcript changes in the spermathecae after mating reflect responses that are likely induced by transferred SFPs.

\section{Conclusions}

We have identified transcriptome responses in female Ae. aegypti reproductive tissues to seminal fluid protein-containing extracts that highlight important functional responses, including immunity, metabolism, 
and protease activity, that take place after mating. We focused on the role of seminal fluid proteins in this response, even though seminal fluid also contains small molecules, metabolites, and RNAs that could potentially also trigger post-mating responses in females, because our earlier work showed that the inducers of post-mating responses were heat-labile, suggesting that they are most likely proteinaceous [22, 29]. The genes identified as responsive to mating that are not responsive to MAG-extract injection could reflect genes that respond to other aspects of mating, such as receipt of sperm or physical contact. It is also possible that we were not able to capture the full extent of DEGs regulated by SFPs. The statistical power to detect smaller, yet significant, transcript changes was hampered by a lower-than-expected number of reads that mapped to genic regions of the $A e$. aegypti reference genome assembly. Introduction of SFPs via intrathoracic injection into the hemocoel, rather than directly into the LRT as in the case of natural mating, could also contribute to an incomplete characterization of all relevant DEGs that respond to SFPs. The transcriptlevel changes identified in this analysis lay the groundwork for further characterization of female genes and associated pathways that are activated by SFPs provided by males. This study represents an important step in understanding the molecular basis of male-female mating interactions that are required for reproductive success and the promotion of post-mating responses elicited by SFPs. These findings provide crucial potential targets for the creation of novel vector control strategies such as ones that target these pathways to disrupt successful reproduction, ultimately reducing vector populations.

\section{Methods}

\section{Mosquitoes}

We used a Thai wild-type strain of Ae. aegypti, established in 2011 and supplemented with field collected mosquitoes from Thailand 1 year prior to our experiments. Mosquitoes were reared as described previously [29].

\section{Preparation and injection of seminal fluid proteins}

Males were anesthetized on ice and their accessory glands (MAG) with seminal vesicles (hereafter referred to as MAG extract) were dissected from 100 five- to sixday-old male adult Ae. aegypti in modified PBS $(137 \mathrm{mM}$ $\mathrm{NaCl}, 2.7 \mathrm{mM} \mathrm{KCl}, 10 \mathrm{mM} \mathrm{Na} \mathrm{HPO}_{4}, 3 \mathrm{mM} \mathrm{KH} \mathrm{KO}_{4}$, $2 \mathrm{mM} \mathrm{CaCl}_{2} \mathrm{pH} 7.0$ ) in a ratio of 1 accessory gland pair per microliter of buffer. Tissue was homogenized with a motorized pestle for ten $1 \mathrm{~s}$ pulses, followed by sonication in a BIORUPTOR (Diagenode, Denville, NJ) at $4{ }^{\circ} \mathrm{C}$ on "high" setting with a cycle of $15 \mathrm{~s}$ on, $15 \mathrm{~s}$ rest, $15 \mathrm{~s}$ on.
After centrifugation at $25,000 \mathrm{~g}$ for $30 \mathrm{~min}$ at $4{ }^{\circ} \mathrm{C}$, the supernatant was recovered and snapfrozen in liquid $\mathrm{N}_{2}$ prior to storage at $-80^{\circ} \mathrm{C}$. All MAG extracts had similar protein concentrations $(1.17 \mu \mathrm{g} / \mu \mathrm{l}, 1.07 \mu \mathrm{g} / \mu \mathrm{l}, 1.05 \mu \mathrm{g} / \mu \mathrm{l})$ as determined with Micro BCA kit (Thermo Fisher Scientific, Waltham, MA). From experiments in which we measured, separately, the protein content of MAGs and seminal vesicles, we estimate that the protein contribution from seminal vesicle was $\sim 5 \%$ of the total lysate. To rule out any contribution of sperm to our results, we stained the extracts with DAPI, to count the number of sperm-heads present. We found only negligible amounts of sperm, comprising $<1$ sperm potentially injected per female. Biological activity in inducing post-mating responses was verified for the MAG extract by injecting them into a subset of virgin females, exposing those females to males 2 days later to provide an opportunity for mating overnight, and dissecting their spermathecae to assess whether or not sperm were present; the latter outcome verified that the extract was active, as it prevented the females from mating. Similarly treated salineinjected females all mated with the males provided.

Virgin females ( 4 to 5 days post-eclosion) were injected intrathoracically with $0.25 \mu \mathrm{l}$ of modified PBS or MAG extract using a Nanoject III (Drummond Scientific Co., Broomall, PA). The amount of injected MAG extract represents 0.25 male accessory gland equivalents, approximately the amount transferred to females during a natural mating $[25,111]$. At $24 \mathrm{~h}$ post-injection, 26 lower reproductive tracts (bursa, spermathecae, and oviducts) were dissected from females in each injection group in chilled PBS and placed immediately into $150 \mu$ l Trizol (Thermo Fisher Scientific, Waltham, MA). Non-injected virgin females $(n=26)$ were dissected as controls (Fig. 1A). All treatments were performed and analyzed in three biological replicates (each with 26 individuals pooled).

\section{RNA isolation and library construction}

Total RNA was extracted with $500 \mu$ l total Trizol following modifications to manufacturer's protocol with utilization of Phaselock gel tubes and GlycoBlue carrier (Thermo Fisher Scientific, Waltham, MA). Following resuspension of the pellet in RNAse-free water, RNA was quantified using a Qubit Broad Range RNA Kit (Thermo Fisher Scientific, Waltham, MA) and quality assessed on an Agilent BioAnalyzer 2100 (Agilent Technologies, Santa Clara, CA). Total RNA (250 ng) was used to prepare libraries using the QuantSeq 3'mRNA library (REV) kit (Lexogen, Greenland, NH). Final libraries were quantified with a Qubit High Sensitivity DNA kit (Thermo Fisher Scientific, Waltham, MA) and quality was assessed on an Agilent BioAnalyzer 2100 (Agilent Technologies, Santa Clara, CA). Sequencing was performed at the 
Cornell Biotechnology Resource Center on a NextSeq500 with the custom sequencing primer (CSP) version 5 supplied by Lexogen (75 bp paired-end reads).

\section{Read processing, alignment, and differential expression analysis}

After checking read quality with FastQC, paired-end reads were aligned to the Ae. aegypti genome (version AaegL5) with Hisat2 using default parameters [112]. In addition to the samples generated in this study, we included in our analysis post-mating RNA-seq samples that were generated in our previous study [46] (Supplementary Fig. 1). Alignments were evaluated with Qualimap [113, 114] and gene counts were extracted using the AaegL5 exon annotations. We detected reads in all replicates and treatments that mapped to intergenic and intronic regions ( 30-40\%) (Supplementary Fig. 3A, 3B). In addition, reads from one of the three non-injected control group library were excluded from the analysis due to a lower alignment rate $(\sim 20 \%$ lower than other samples) and higher read pair discordance (3\% higher than other samples) (Supplementary Fig. 3C, 3D). Additionally, replicate analysis before RUVSeq batch correction found this sample to be an outlier with respect to the grouping of the other samples and expression profile Pearson correlation coefficients (Supplementary Fig. 3E, $3 \mathrm{~F})$.

The gene counts matrix was filtered to remove genes with low-abundance RNAs $(\mathrm{CPM}<5)$. For differential abundance analysis, we controlled for sources of erroneous variation using RUVseq with $k=3$ [115] then fit a quasi-likelihood generalized linear model that incorporates the RUVseq residuals to the count data using edgeR [116] (Supplementary Fig. 4). Replicates grouped together as determined via multi-dimensional scaling (Fig. 1B). The differential expression analysis involved setting up the following contrasts at $24 \mathrm{~h}$ post-injection: (1) between the saline-injected and non-injected samples, (2) between the MAG extract-injected and noninjected samples, and (3) between MAG extract-injected and saline-injected samples (Supplementary Fig. 5). We defined genes as significantly differentially expressed between treatments if transcript abundance was $>2$-fold with a Benjamini-Hochberg corrected false-discovery rate $(\mathrm{FDR})<0.05$ [117]. Identified DEGs from the comparison of MAG extract-injected and non-injected samples were further compared to DEGs identified in the LRT or spermathecae $24 \mathrm{~h}$ after mating $[46,57]$. Functional annotation resources were acquired from VectorBase (vectorbase.org) and were generated previously [16]. Gene Ontology (GO) enrichment analysis was carried out using GOSeq [118]. For GeneIDs not characterized in VectorBase, we searched for predicted conserved domains with NCBI Conserved Domain database [119] or similar genes by tBLASTn [120].

\section{Supplementary Information}

The online version contains supplementary material available at https://doi. org/10.1186/s12864-021-08201-0.

Additional file 1. Supplementary Tables 1-3 and Figures 1-5.

Additional file 2. Supplementary File 1-Mated vs virgin updated analysis.

\section{Acknowledgements}

We thank Kevin Pritts for assistance with mosquito rearing, Jen Grenier from the Cornell Transcriptional Regulation and Expression facility for providing valuable advice on RNA extraction, Bretta Hixson and Nicolas Buchon for reagents, Julián Hillyer for helpful discussions, and the team at the Cornell Biotechnology Resource Center for sequencing assistance.

\section{Authors' contributions}

I.A.A., M.F.W., and L.C.H., conceived and designed experiments. G.P.L., S.P., F.W.A., and I.A.A. conducted experiments to generate libraries. Y.H.A.-B. and I.A.A. analyzed and interpreted results. P.C.C. aided in figure design. I.A.A., M.F.W., and L.C.H wrote the manuscript. All authors reviewed and approved the final manuscript.

\section{Funding}

This work was made possible by support from NIH/NIAID R01-AI095491 to L.C.H. and M.F.W., and COLCIENCIAS-Universidad de Antioquia and the Max Planck Society cooperation Grant 566-1 (2014) to F.W.A.. For part of this work, I. A. A. was supported by Training Grant T32HD052471 from the National Institutes of Child Health and Human Development. The content is solely the responsibility of the authors and does not necessarily represent the official views of the National Institute of Child Health and Human Development.

\section{Availability of data and materials}

The Illumina sequence reads are available through the Sequence Read Archive (SRA) under project accession PRJNA701279. The processed data files and analysis scripts are available through a GitHub repository: https://github.com/ YazBraimah/Aegypti_MAG_Saline_RNAseq.

\section{Declarations}

Ethics approval and consent to participate Not Applicable.

\section{Consent for publication}

Not Applicable.

\section{Competing interests}

The authors declare no competing interests.

\section{Author details}

${ }^{1}$ Department of Entomology, Cornell University, Ithaca, NY 14853, USA. ${ }^{2}$ Department of Biology, Syracuse University, Syracuse, NY 13244, USA. ${ }^{3}$ Max Planck Tandem Group in Mosquito Reproductive Biology, Universidad de Antioquia, Medellín 050010, Colombia. ${ }^{4}$ Department of Molecular Biology and Genetics, Cornell University, Ithaca, NY 14853, USA.

Received: 9 August 2021 Accepted: 23 November 2021

Published online: 15 December 2021

\section{References}

1. Cunha MS, Costa PAG, Correa IA, de Souza MRM, Calil PT, da Silva GPD, et al. Chikungunya virus: an emergent arbovirus to the south American 
continent and a continuous threat to the world. Front Microbiol. 2020;11:1297.

2. Monath TP, Vasconcelos PFC. Yellow fever. J Clin Virol. 2015;64:160-73.

3. Pielnaa P, Al-Saadawe M, Saro A, Dama MF, Zhou M, Huang Y, et al. Zika virus-spread, epidemiology, genome, transmission cycle, clinical manifestation, associated challenges, vaccine and antiviral drug development. Virology. 2020;543:34-42.

4. Harapan H, Michie A, Sasmono RT, Imrie A. Dengue: a Minireview. Viruses. 2020;12:829 Multidisciplinary Digital Publishing Institute.

5. de Godoy AS, Sachetto Fernandes R, Campos Aguiar AC, Vieira Bueno R, de Moraes Roso Mesquita NC, Carvalho Guido RV, et al. Structural and mechanistic insight from antiviral and antiparasitic enzyme drug targets for tropical infectious diseases. Curr Opin Struct Biol. 2019;59:65-72.

6. Troost B, Smit JM. Recent advances in antiviral drug development towards dengue virus. Curr Opin Virol. 2020;43:9-21.

7. Chen LH, Wilson ME. Yellow fever control: current epidemiology and vaccination strategies. Trop Dis Travel Med Vaccines. 2020;6:1-10 BioMed Central.

8. Pinheiro-Michelsen JR, Souza RDSO, Santana IVR, da Silva P d S, Mendez EC, Luiz WB, et al. Anti-dengue vaccines: from development to clinical trials. Front Immunol Front. 2020;11:1252.

9. Dusfour I, Vontas J, David J-P, Weetman D, Fonseca DM, Corbel V, et al. Management of insecticide resistance in the major Aedes vectors of arboviruses: advances and challenges. Fuehrer H-P, editor. PLoS Negl Trop Dis. 2019;13:e0007615 Public Library of Science.

10. Ginsberg HS, Bargar TA, Hladik ML, Lubelczyk C. Management of Arthropod Pathogen Vectors in North America: minimizing adverse effects on pollinators. J Med Entomol. 2017:54(6):1463-75.

11. Macias VM, Ohm JR, Rasgon JL. Gene drive for mosquito control: where did it come from and where are we headed? Int I Environ Res Public Health. 2017;14:1006 Multidisciplinary Digital Publishing Institute.

12. Cator LJ, Wyer CAS, Harrington LC. Mosquito sexual selection and reproductive control programs. Trends Parasitol. 2021;32:207.

13. Avila FW, Sirot LK, LaFlamme BA, Rubinstein CD, Wolfner MF. Insect seminal fluid proteins: identification and function. Annu. Rev. Entomol. Annu Rev. 2011:56:21-40.

14. Klowden MJ. The check is in the male: male mosquitoes affect female physiology and behavior. J Am Mosq Control Assoc. 1999;15:213-20.

15. Baldini F, Gabrieli P, Rogers DW, Catteruccia F. Function and composition of male accessory gland secretions in Anopheles gambiae: a comparison with other insect vectors of infectious diseases. Pathog Glob Health. 2012;106:82-93.

16. Degner EC, Ahmed-Braimah YH, Borziak K, Wolfner MF, Harrington LC, Dorus S. Proteins, transcripts, and genetic architecture of seminal fluid and sperm in the mosquito Aedes aegypti. Mol Cell Proteomics. 2019;18:S6-S22.

17. Wigby S, Brown NC, Allen SE, Misra S, Sitnik JL, Sepil I, et al. The Drosophila seminal proteome and its role in postcopulatory sexual selection. Philos Trans R Soc Lond Ser B Biol Sci. 2020;375:20200072 The Royal Society.

18. Judson C. Feeding and oviposition behavior in the mosquito Aedes aegypti (L.). I. preliminary studies of physiological control mechanisms. Biol Bull. 1967;133:369-77.

19. Wallis RC, Lang C. Egg formation and oviposition in blood-fed Aedes aegypti L. Mosquito News. 1956;16:283-6.

20. Lang $C$. The influence of mating on egg production by Aedes aegypti. Am. J. Trop. Med. Hyg. 1956;5:909-14.

21. Klowden MJ, Chambers GM. Male accessory gland substances activate egg development in nutritionally stressed Aedes aegypti mosquitoes. J Insect Physiol. 1991;37:721-6 Pergamon.

22. Villarreal SM, Pitcher S, Helinski MEH, Johnson L, Wolfner MF, Harrington $L C$. Male contributions during mating increase female survival in the disease vector mosquito Aedes aegypti. J Insect Physiol. 2018;108:1-9.

23. Helinski MEH, Harrington LC. Male mating history and body size influence female fecundity and longevity of the dengue vector Aedes aegypti. J Med Entomol. 2011;48:202-11.
24. Craig GB. Mosquitoes: female monogamy induced by male accessory gland substance. Science. 1967;156:1499-501 American Association for the Advancement of Science.

25. Helinski MEH, Deewatthanawong P, Sirot LK, Wolfner MF, Harrington LC. Duration and dose-dependency of female sexual receptivity responses to seminal fluid proteins in Aedes albopictus and Ae. aegypti mosquitoes. J Insect Physiol. 2012;58:1307-13.

26. Degner EC, Harrington LC. Polyandry depends on postmating time interval in the dengue vector Aedes aegypti. Am J Trop Med Hyg. 2016;94:780-5 American Society of Tropical Medicine and Hygiene.

27. Fuchs MS, Craig GB, Hiss EA. The biochemical basis of female monogamy in mosquitoes. I. Extraction of the active principle from Aedes aegypti. Life Sci. 1968;7:835-9.

28. Duvall LB, Basrur NS, Molina H, McMeniman CJ, Vosshall LB. A peptide signaling system that rapidly enforces paternity in the Aedes aegypti Mosquito. Curr Biol. 2017;27:3734-5.

29. League GP, Baxter LL, Wolfner MF, Harrington LC. Male accessory gland molecules inhibit harmonic convergence in the mosquito Aedes aegypti. Curr Biol. 2019;29:R196-7.

30. Lawniczak MKN, Begun DJ. A genome-wide analysis of courting and mating responses in Drosophila melanogaster females. Genome. 2004:47:900-10.

31. McGraw LA, Gibson G, Clark AG, Wolfner MF. Genes regulated by mating, sperm, or seminal proteins in mated female Drosophila melanogaster. Curr Biol. 2004;14:1509-14.

32. Kocher SD, Richard F-J, Tarpy DR, Grozinger CM. Genomic analysis of post-mating changes in the honeybee queen (Apis mellifera). BMC Genomics. 2008;9:232-15 BioMed Central.

33. McGraw LA, Clark AG, Wolfner MF. Post-mating gene expression profiles of female Drosophila melanogaster in response to time and to four male accessory gland proteins. Genetics. 2008;179:1395-408.

34. Rogers DW, Whitten MMA, Thailayil J, Soichot J, Levashina EA, Catteruccia F. Molecular and cellular components of the mating machinery in Anopheles gambiae females. Proc Natl Acad Sci. 2008;105:19390-5 U.S.A. National Academy of Sciences.

35. Bono JM, Matzkin LM, Kelleher ES, Markow TA. Postmating transcriptional changes in reproductive tracts of con- and heterospecifically mated Drosophila mojavensis females. Proc Natl Acad Sci. 2011;108:7878-83 U.S.A. National Academy of Sciences.

36. Gomulski LM, Dimopoulos G, Xi Z, Scolari F, Gabrieli P, Siciliano P, et al. Transcriptome profiling of sexual maturation and mating in the Mediterranean fruit fly, Ceratitis capitata. Ouzounis CA, editor. PLoS One. 2012; 7:e30857 Public Library of Science.

37. Short SM, Lazzaro BP. Reproductive status alters transcriptomic response to infection in female Drosophila melanogaster. G3 (Bethesda) G3: Genes, Genomes, Genet. 2013;3:827-40.

38. Al-Wathiqui N, Lewis SM, Dopman EB. Using RNA sequencing to characterize female reproductive genes between $Z$ and $E$ strains of European corn borer moth (Ostrinia nubilalis). BMC Genomics. 2014;15:189-13 BioMed Central.

39. Zheng W, Luo D, Wu F, Wang J, Zhang H. RNA sequencing to characterize transcriptional changes of sexual maturation and mating in the female oriental fruit fly Bactrocera dorsalis. BMC Genomics. 2016;17:19413 2nd ed. BioMed Central.

40. Immonen E, Sayadi A, Bayram H, Arnqvist G. Mating changes sexually dimorphic gene expression in the seed beetle Callosobruchus maculatus. Genome Biol Evol. 2017;9:677-99.

41. Kumaran N, van der Burg CA, Qin Y, Cameron SL, Clarke AR, Prentis PJ. Plant-mediated female transcriptomic changes post-mating in a Tephritid fruit Fly, Bactrocera tryoni. Genome Biol Evol. 2018;10:94-107.

42. Crava CM, Sassù F, Tait G, Becher PG, Anfora G. Functional transcriptome analyses of Drosophila suzukii antennae reveal mating-dependent olfaction plasticity in females. Insect Biochem Mol Biol. 2019;105:51-9.

43. Liu P-C, Hao D-J. Behavioural and transcriptional changes in postmating females of an egg parasitoid wasp species. R Soc Open Sci. 2019;6:181453 The Royal Society.

44. Huo Z, Liu Y, Yang J, Xie W, Wang S, Wu Q, et al. Transcriptomic analysis of mating responses in Bemisia tabaci MED females. Insects. 2020;11:308 Multidisciplinary Digital Publishing Institute. 
45. Ahmed-Braimah YH, Wolfner MF, Clark AG. Differences in post-mating transcriptional responses between conspecific and heterospecific matings in Drosophila. Mol Biol Evol. 2020;6:4067.

46. Alfonso-Parra C, Ahmed-Braimah YH, Degner EC, Avila FW, Villarreal SM, Pleiss JA, et al. Mating-induced transcriptome changes in the reproductive tract of female Aedes aegypti. Benedict MQ, editor. PLoS Negl Trop Dis. 2016;10:e0004451 Public Library of Science.

47. Mack PD, Kapelnikov A, Heifetz Y, Bender M. Mating-responsive genes in reproductive tissues of female Drosophila melanogaster. Proc Natl Acad Sci. 2006;103:10358-63 U.S.A. National Academy of Sciences.

48. Kapelnikov A, Zelinger E, Gottlieb Y, Rhrissorrakrai K, Gunsalus KC, Heifetz Y. Mating induces an immune response and developmental switch in the Drosophila oviduct. Proc Natl Acad Sci. 2008;105:13912-7 U.S.A. National Academy of Sciences.

49. Gabrieli P, Kakani EG, Mitchell SN, Mameli E, Want EJ, Mariezcurrena Anton A, et al. Sexual transfer of the steroid hormone 20E induces the postmating switch in Anopheles gambiae. Proc Natl Acad Sci. 2014:111:16353-8 U.S.A. National Academy of Sciences.

50. McDonough-Goldstein CE, Borziak K, Pitnick S, Dorus S. Drosophila female reproductive tract gene expression reveals coordinated mating responses and rapidly evolving tissue-specific genes. G3 (Bethesda). 2021;11(3):jkab020.

51. Thailayil J, Magnusson K, Godfray HCJ, Crisanti A, Catteruccia F. Spermless males elicit large-scale female responses to mating in the malaria mosquito Anopheles gambiae. Proc Natl Acad Sci. 2011;108:13677-81 U.S.A. National Academy of Sciences.

52. Gabrieli P, Scolari F, Di Cosimo A, Savini G, Fumagalli M, Gomulski LM, et al. Sperm-less males modulate female behaviour in Ceratitis capitata (Diptera: Tephritidae). Insect Biochem Mol Biol. 2016;79:13-26.

53. Shaw WR, Teodori E, Mitchell SN, Baldini F, Gabrieli P, Rogers DW, et al. Mating activates the heme peroxidase HPX15 in the sperm storage organ to ensure fertility in Anopheles gambiae. Proc Natl Acad Sci. 2014;111:5854-9 U.S.A. National Academy of Sciences.

54. Lung O, Wolfner MF. Drosophila seminal fluid proteins enter the circulatory system of the mated female fly by crossing the posterior vaginal wall. Insect Biochem Mol Biol. 1999;29:1043-52.

55. Mattei AL, Riccio ML, Avila FW, Wolfner MF. Integrated 3D view of postmating responses by the Drosophila melanogaster female reproductive tract, obtained by micro-computed tomography scanning. Proc Natl Acad Sci. 2015;112:8475-80 U.S.A. National Academy of Sciences.

56. Chen PS, Stumm-Zollinger E, Caldelari M. Protein metabolism of Drosophila male accessory glands-II: species-specificity of secretion proteins. Insect Biochem. 1985;15:385-90 Pergamon.

57. Camargo C, Ahmed-Braimah YH, Amaro IA, Harrington LC, Wolfner MF, Avila FW. Mating and blood-feeding induce transcriptome changes in the spermathecae of the yellow fever mosquito Aedes aegypti 2 nd ed. Sci Rep. 2020;10:14899-13 Nature Publishing Group.

58. Waterhouse RM, Kriventseva EV, Meister S, Xi Z, Alvarez KS, Bartholomay LC, et al. Evolutionary dynamics of immune-related genes and pathways in disease-vector mosquitoes. Science. 2007;316:1738-43 American Association for the Advancement of Science.

59. Hillyer JF. Mosquito immunity. Adv Exp Med Biol. 2010;708:218-38 8 ed. Boston, MA: Springer US.

60. Christensen BM, Li J, Chen C-C, Nappi AJ. Melanization immune responses in mosquito vectors. Trends Parasitol. 2005;21:192-9.

61. Johnson JK, Li J, Christensen BM. Cloning and characterization of a dopachrome conversion enzyme from the yellow fever mosquito, Aedes aegypti. Insect Biochem Mol Biol. 2001;31:1125-35.

62. Kanost MR, Jiang H. Clip-domain serine proteases as immune factors in insect hemolymph. Curr Opin Insect Sci. 2015;11:47-55.

63. Meekins DA, Kanost MR, Michel K. Serpins in arthropod biology. Semin Cell Dev Biol. 2017:62:105-19.

64. Moussawi El L, Nakhleh J, Kamareddine L, Osta MA. The mosquito melanization response requires hierarchical activation of non-catalytic clip domain serine protease homologs. Dimopoulos G, editor. PLoS Pathog. 2019:15:e1008194 Public Library of Science.

65. Theopold U, Schmidt O, Söderhäll K, Dushay MS. Coagulation in arthropods: defence, wound closure and healing. Trends Immunol. 2004;25:289-94.

66. Mylonakis E, Podsiadlowski L, Muhammed M, Vilcinskas A. Diversity, evolution and medical applications of insect antimicrobial peptides
Philos Trans R Soc Lond Ser B Biol Sci. 2016;371:20150290 The Royal Society.

67. Kolliopoulou A, Santos D, Taning CNT, Wynant N, Vanden Broeck J, Smagghe G, et al. PIWI pathway against viruses in insects. Wiley Interdiscip Rev RNA. 2019;10:e1555 John Wiley \& Sons, Ltd.

68. Vargas V, Moreno-García M, Duarte-Elguea E, Lanz-Mendoza H. Limited specificity in the injury and infection priming against Bacteria in Aedes aegypti mosquitoes. Front Microbiol Front. 2016;7:975.

69. Spray FJ, Christensen BM. Aedes aegypti: characterization of hemocyte polypeptide synthesis during wound healing and immune response to inoculated microfilariae. Exp Parasitol. 1991;73:481-8.

70. Ramond E, Dudzic JP, Lemaitre B. Comparative RNA-Seq analyses of Drosophila plasmatocytes reveal gene specific signatures in response to clean injury and septic injury. Bergmann A, editor. PLoS One. 2020;15:e0235294 Public Library of Science.

71. Márkus R, Kurucz E, Rus F, Andó I. Sterile wounding is a minimal and sufficient trigger for a cellular immune response in Drosophila melanogaster. Immunol Lett. 2005;101:108-11.

72. Lai SC, Chen CC, Hou RF. Electron microscopic observations on wound-healing in larvae of the mosquito Armigeres subalbatus (Diptera: Culicidae). J Med Entomol. 2001;38:836-43.

73. Abbas MN, Kausar S, Zhao E, Cui H. Suppressors of cytokine signaling proteins as modulators of development and innate immunity of insects. Dev Comp Immunol. 2020;104:103561.

74. Matthews BJ, Dudchenko O, Kingan SB, Koren S, Antoshechkin I, Crawford JE, et al. Improved reference genome of Aedes aegypti informs arbovirus vector control. Nature. 2018;563:501-7 Nature Publishing Group.

75. Peng J, Zipperlen P, Kubli E. Drosophila sex-peptide stimulates female innate immune system after mating via the toll and Imd pathways. Curr Biol. 2005:15:1690-4.

76. Short SM, Wolfner MF, Lazzaro BP. Female Drosophila melanogaster suffer reduced defense against infection due to seminal fluid components. J Insect Physiol. 2012;58:1192-201.

77. Delbare SYN, Ahmed-Braimah YH, Wolfner MF, Clark AG. Interactions between the microbiome and mating influence the female's transcriptional profile in Drosophila melanogaster. Sci Rep. 2020;10:18168-12 Nature Publishing Group.

78. Delbare SYN, Chow CY, Wolfner MF, Clark AG. Roles of female and male genotype in post-mating responses in Drosophila melanogaster. J Hered. 2017; 108:740-53.

79. Baer B, Armitage SAO, Boomsma JJ. Sperm storage induces an immunity cost in ants. Nature. 2006;441:872-5 Nature Publishing Group.

80. Lawniczak MKN, Barnes Al, Linklater JR, Boone JM, Wigby S, Chapman T. Mating and immunity in invertebrates. Trends Ecol Evol (Amst). 2007:22:48-55.

81. Schwenke RA, Lazzaro BP, Wolfner MF. Reproduction-immunity tradeoffs in insects. Annu Rev Entomol Annu Rev. 2016;61:239-56.

82. Oku K, Price TAR, Wedell N. Does mating negatively affect female immune defences in insects? Anim Biol. 2019;69:117-36.

83. Wigby S, Suarez SS, Lazzaro BP, Pizzari T, Wolfner MF. Sperm success and immunity. Curr Top Dev Biol. 2019;135:287-313 Elsevier.

84. Li H-H, Cai Y, Li J-C, Su MP, Liu W-L, Cheng L, et al. C-type lectins link immunological and reproductive processes in Aedes aegypti. iscience. 2020;23:101486.

85. Pompon J, Levashina EA. A new role of the mosquito complement-like cascade in male fertility in Anopheles gambiae. Schneider DS, editor. PLoS Biol. 2015;13:e1002255.

86. LaFlamme BA, Wolfner MF. Identification and function of proteolysis regulators in seminal fluid. Mol Reprod Dev. 2013;80:80-101 John Wiley \& Sons, Ltd.

87. Degner EC, Harrington LC. A mosquito sperm's journey from male ejaculate to egg: mechanisms, molecules, and methods for exploration. Mol Reprod Dev. 2016:83:897-911 John Wiley \& Sons, Ltd.

88. Stephens K, Cardullo RA, Thaler CD. Culex pipiens sperm motility is initiated by a trypsin-like protease from male accessory glands. Mol Reprod Dev. 2018;85:440-8.

89. LaFlamme BA, Ram KR, Wolfner MF. The Drosophila melanogaster seminal fluid protease "seminase" regulates proteolytic and post-mating reproductive processes. PLoS Genet. 2012;8:e1002435 Public Library of Science. 
90. Bascuñán P, Gabrieli P, Mameli E, Catteruccia F. Mating-regulated atrial proteases control reinsemination rates in Anopheles gambiae females. Sci Rep. 2020;10:21974-10 Nature Publishing Group.

91. Lindsay LL, Wieduwilt MJ, Hedrick JL. Oviductin, the Xenopus laevis oviductal protease that processes egg envelope glycoprotein gp43, increases sperm binding to envelopes, and is translated as part of an unusual mosaic protein composed of two protease and several CUB domains. Biol Reprod. 1999;60:989-95.

92. Hiyoshi M, Takamune K, Mita K, Kubo H, Sugimoto Y, Katagiri C. Oviductin, the oviductal protease that mediates gamete interaction by affecting the vitelline coat in Bufo japonicus: its molecular cloning and analyses of expression and posttranslational activation. Dev Biol. 2002;243:176-84.

93. Saurabh S, Vanaphan N, Wen W, Dauwalder B. High functional conservation of takeout family members in a courtship model system. Hansen IA, editor. PLoS One. 2018;13:e0204615 Public Library of Science.

94. Zhou J-J, He X-L, Pickett JA, Field LM. Identification of odorantbinding proteins of the yellow fever mosquito Aedes aegypti: genome annotation and comparative analyses. Insect Mol Biol. 2008;17:14763 John Wiley \& Sons, Ltd.

95. Matthews BJ, McBride CS, DeGennaro M, Despo O, Vosshall LB. The neurotranscriptome of the Aedes aegypti mosquito. BMC Genomics. 2016;17:32-20 BioMed Central.

96. Tallon AK, Hill SR, Ignell R. Sex and age modulate antennal chemosensory-related genes linked to the onset of host seeking in the yellow-fever mosquito, Aedes aegypti. Sci Rep. 2019;9:43-13 Nature Publishing Group.

97. Pelosi P, lovinella I, Zhu J, Wang G, Dani FR. Beyond chemoreception: diverse tasks of soluble olfactory proteins in insects. Biol Rev Camb Philos Soc. 2018;93:184-200 John Wiley \& Sons, Ltd.

98. Song Y-Q, Song Z-Y, Dong J-F, Lv Q-H, Chen Q-X, Sun H-Z. Identification and comparative expression analysis of odorant-binding proteins in the reproductive system and antennae of Athetis dissimilis. Sci Rep. 2021;11:13941-13 Nature Publishing Group.

99. Findlay GD, Yi X, Maccoss MJ, Swanson WJ. Proteomics reveals novel Drosophila seminal fluid proteins transferred at mating. PLoS Biol. 2008;6:e178 Public Library of Science.

100. Rihani K, Ferveur J-F, Briand L. The 40-year mystery of insect odorantbinding proteins. Biomolecules. 2021;11:509 Multidisciplinary Digital Publishing Institute.

101. Zhang DB, Wang Y, Liu AK, Wang XH, Dang CW, Yao Q, et al. Phylogenetic analyses of vector mosquito basic helix-loop-helix transcription factors. Insect Mol Biol. 2013;22:608-21 John Wiley \& Sons, Ltd.

102. Bitra K, Palli SR. The members of bHLH transcription factor superfamily are required for female reproduction in the red flour beetle, Tribolium castaneum. J Insect Physiol. 2010;56:1481-9.

103. Chaharbakhshi E, Jemc JC. Broad-complex, tramtrack, and bric-àbrac (BTB) proteins: critical regulators of development. Genesis. 2016:54:505-18.

104. Mukai M, Hayashi Y, Kitadate Y, Shigenobu S, Arita K, Kobayashi S. MAMO, a maternal BTB/POZ-Zn-finger protein enriched in germline progenitors is required for the production of functional eggs in Drosophila. Mech Dev. 2007:124:570-83.

105. Jasper WC, Brutscher LM, Grozinger CM, Niño EL. Injection of seminal fluid into the hemocoel of honeybee queens (Apis mellifera) can stimulate post-mating changes. Sci Rep. 2020;10:11990-18 Nature Publishing Group.

106. Farley EJ, Eggleston $\mathrm{H}$, Riehle MM. Filtering the junk: assigning function to the mosquito non-coding genome. Insects. 2021;12:186 Multidisciplinary Digital Publishing Institute.

107. Statello L, Guo C-J, Chen L-L, Huarte M. Gene regulation by long non-coding RNAs and its biological functions. Nat Rev Mol Cell Biol. 2021;22:96-118 Nature Publishing Group.

108. Pascini TV, Ramalho-Ortigão M, Ribeiro JM, Jacobs-Lorena M, Martins GF. Transcriptional profiling and physiological roles of Aedes aegypti spermathecal-related genes. BMC Genomics. 2020;21:143-18 BioMed Central.

109. Prokupek AM, Kachman SD, Ladunga I, Harshman LG. Transcriptional profiling of the sperm storage organs of Drosophila melanogaster. Insect Mol Biol. 2009;18:465-75.
110. Gotoh A, Shigenobu S, Yamaguchi K, Kobayashi S, Ito F, Tsuji K. Transcriptome profiling of the spermatheca identifies genes potentially involved in the long-term sperm storage of ant queens. Sci Rep. 2017;7:5972-14 Nature Publishing Group.

111. Alfonso-Parra C, Avila FW, Deewatthanawong P, Sirot LK, Wolfner MF, Harrington LC. Synthesis, depletion and cell-type expression of a protein from the male accessory glands of the dengue vector mosquito Aedes aegypti. J Insect Physiol. 2014;70:117-24.

112. Kim D, Langmead B, Salzberg SL. HISAT: a fast spliced aligner with low memory requirements. Nat Methods. 2015;12:357-60 Nature Publishing Group.

113. García-Alcalde F, Okonechnikov K, Carbonell J, Cruz LM, Götz S, Tarazona S, et al. Qualimap: evaluating next-generation sequencing alignment data. Bioinformatics. 2012;28:2678-9.

114. Okonechnikov K, Conesa A, García-Alcalde F. Qualimap 2: advanced multi-sample quality control for high-throughput sequencing data. Bioinformatics. 2016;32:292-4.

115. Risso D, Ngai J, Speed TP, Dudoit S. Normalization of RNA-seg data using factor analysis of control genes or samples. Nat Biotechnol. 2014;32:896-902 Nature Publishing Group.

116. Robinson MD, McCarthy DJ, Smyth GK. edgeR: a Bioconductor package for differential expression analysis of digital gene expression data. Bioinformatics. 2010;26:139-40.

117. Benjamini Y, Hochberg Y. Multiple hypotheses testing with weights. Scand J Stat. 1997;24:407-18.

118. Young MD, Wakefield MJ, Smyth GK, Oshlack A. Gene ontology analysis for RNA-seq: accounting for selection bias. Genome Biol. 2010;11:R14-2 BioMed Central.

119. Lu S, Wang J, Chitsaz F, Derbyshire MK, Geer RC, Gonzales NR, et al. CDD/SPARCLE: the conserved domain database in 2020. Nucleic Acids Res. 2020:48:D265-8.

120. Johnson M, Zaretskaya I, Raytselis Y, Merezhuk Y, McGinnis S, Madden TL. NCBI BLAST: a better web interface. Nucleic Acids Res. 2008;36:W5-9.

\section{Publisher's Note}

Springer Nature remains neutral with regard to jurisdictional claims in published maps and institutional affiliations.

Ready to submit your research? Choose BMC and benefit from

- fast, convenient online submission

- thorough peer review by experienced researchers in your field

- rapid publication on acceptance

- support for research data, including large and complex data types

- gold Open Access which fosters wider collaboration and increased citations

- maximum visibility for your research: over $100 \mathrm{M}$ website views per year

At BMC, research is always in progress.

Learn more biomedcentral.com/submissions 